\title{
PAH growth in flames and space: Formation of phenalenyl radical
}

\author{
Zachariah D. Levey, ${ }^{\dagger}$ Benjamin A. Laws,${ }^{\dagger}$ Srivathsan P. Sundar,$\ddagger$ \\ Klaas Nauta, ${ }^{\dagger}$ Scott H. Kable,${ }^{\dagger}$ Gabriel da Silva, ${ }^{\ddagger}$ John F. Stanton, ${ }^{\uparrow}$ and \\ Timothy W. Schmidt*, ${ }^{*}$ \\ $\dagger$ School of Chemistry, University of New South Wales, Sydney NSW 2052, Australia \\ $\ddagger$ Department of Chemical Engineering, The University of Melbourne, Parkville 3010, Australia \\ 9Department of Chemistry, University of Florida, Gainesville, Florida 32611, USA \\ $\S$ Centre of Excellence in Exciton Science, University of New South Wales, Sydney NSW 2052, \\ Australia \\ E-mail: timothy.schmidt@unsw.edu.au
}

\begin{abstract}
Polycyclic aromatic hydrocarbons (PAHs) are intermediates in the formation of soot particles and interstellar grains. However, their formation mechanisms in combustion and interstellar environments are not fully understood. The production of tricyclic PAHs and, in particular, the conversion of a PAH containing a five-membered ring to one with a six-membered ring is of interest to explain PAH abundances in combustion processes. In the present work, resonant ionization mass spectrometry in conjunction with isotopic labelling is used to investigate the formation of the phenalenyl radical from acenaphthylene and methane in an electrical discharge. We show that in this environment, the $\mathrm{CH}$ cycloaddition mechanism converts a fivemembered ring to a six-membered ring. This mechanism can occur in tandem with other PAH
\end{abstract}


formation mechanisms such as hydrogen abstraction/ acetylene addition (HACA) to produce larger PAHs in flames and the interstellar medium.

\section{Introduction}

Polycyclic aromatic hydrocarbons (PAHs) are early intermediates in the soot formation process in both terrestrial ${ }^{1-3}$ and interstellar environments ${ }^{4-7}$. Soot and PAHs formed during the incomplete combustion of fossil fuels are detrimental to human health due to their toxic and carcinogenic properties $^{8-11}$. A ubiquitous existence of PAHs in the interstellar medium (ISM) is inferred from the aromatic infrared bands (AIBs), a set of infrared emission features between $3-20 \mu \mathrm{m}$ that are characteristic of large PAH molecules ${ }^{12,13}$. It is thought that PAHs may comprise up to $20 \%$ of the total cosmic carbon abundance ${ }^{14-16}$. Benzene and benzonitrile, as well as fullerenes have been identified in the ISM ${ }^{17-20}$. However, the detection of any specific PAH had been elusive, until recently, when the emission from 1- and 2- cyanonaphthalene was observed within the Taurus Molecular Cloud, TMC-1 ${ }^{21}$.

The formation mechanism for interstellar PAHs is ambiguous. Astrochemical models of PAH formation are derived from combustion chemistry models ${ }^{22}$. Bottom-up molecular growth processes in circumstellar envelopes of carbon-rich stars are largely based on the hydrogen abstractionacetylene addition (HACA) mechanism ${ }^{12}$. HACA proceeds by activation of a radical site by $\mathrm{H}$ abstraction or addition, followed by addition of acetylene and ring closure ${ }^{23}$. It has been shown to occur for the formation of naphthalene from benzene via a phenylacetylene intermediate ${ }^{24,25}$, as well as for the formation of pyrene from 4-phenanthrenyl radical ${ }^{26}$.

However, there are some shortcomings of the HACA mechanism. Notably, modelling studies have reported that HACA under-predicts the concentration of PAHs in flames ${ }^{27}$, and under-predicts the PAH growth rate in astrochemical models ${ }^{28-30}$. To address these deficiencies, additional PAH formation mechanisms have been proposed. These include: recombination of resonance-stabilized radicals $^{31-33}$; dimerization of small PAHs ${ }^{34}$; phenyl addition/cyclization; ${ }^{35}$ methyl addition/cyclization ${ }^{36}$, 
and methylidyne addition-cyclization-aromatization (MACA) ${ }^{37}$.

Acenaphthylene (ACYN) is considered the first island of stability pulling the HACA sequence forward ${ }^{38}$. Theoretical investigations of the reaction between naphthalene and acetylene show predominant formation of $\mathrm{ACYN}$ over phenanthrene and anthracene: rapid cyclization after the addition of a single acetylene adjacent to the bay region to form a five-membered ring occurs faster than the addition of a second acetylene, followed by cyclization to form a third aromatic ring ${ }^{39}$. This mechanism is depicted in Figure 1a. Indeed, three-quarters of the PAH products of the reaction contain a five-membered ring and less than $6 \%$ are PAHs with exclusively six-membered rings ${ }^{39}$. Hence, a mechanism to convert a five-membered ring on the edge of a PAH to a sixmembered ring is necessary to account for pathways to larger PAHs.

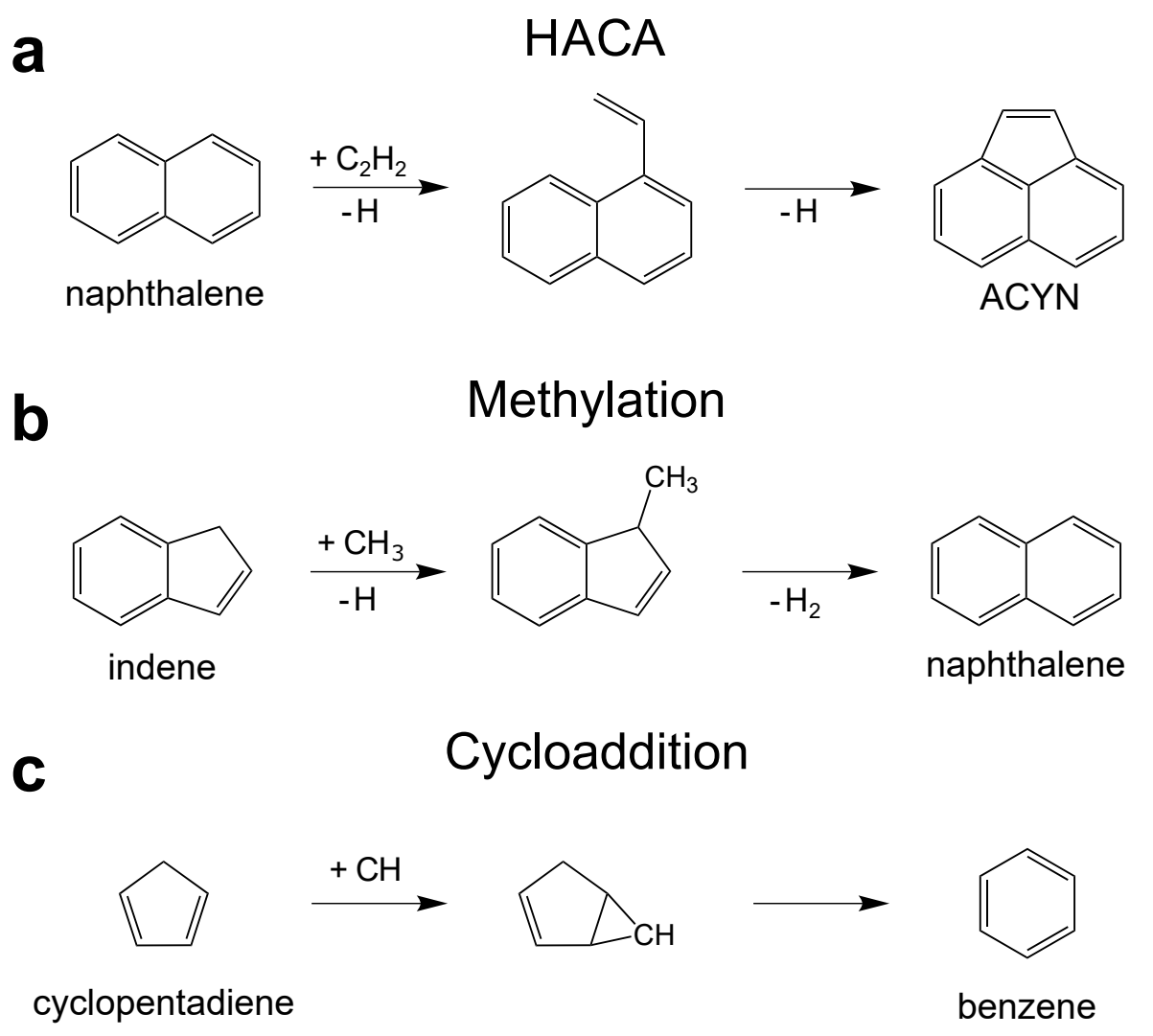

Figure 1: Proposed mechanisms for PAH growth. a) Formation of ACYN from naphthalene via HACA. b) Formation of naphthalene from indene via methylation. c) Formation of benzene from cyclopentadiene via cycloaddition.

Ring expansion has been proposed to take place by methylation followed by rearrangement and 
H-loss, and this mechanism has been invoked to account for benzene formation from cyclopentadienyl radical and naphthalene formation from indene (Figure 1b) ${ }^{31,40-45}$ Recently, calculations for the reaction of 1-acenaphthyl and methyl suggest that ring expansion of the five-membered ring occurs by methylation to produce $1 \mathrm{H}$-phenalene and the phenalenyl radical ${ }^{46}$. Phenalenyl radical $^{47,48}$ is posited to play a role in soot inception and growth ${ }^{33}$. It consists of three aromatic rings which share a central carbon atom. The gas phase excitation spectrum for the $\mathrm{D}_{1} \leftarrow \mathrm{D}_{0}$ electronic transition was reported by our group ${ }^{49}$.

Additionally, expansion of a five-membered ring to a six-membered ring has been demonstrated through the reaction of pyrrole with the methylidyne radical $(\mathrm{CH})$ to form pyridine ${ }^{50}$, and the reaction of cyclopentadiene with $\mathrm{CH}$ to form benzene (Figure 1c ${ }^{51,52}$. $\mathrm{CH}$ in its ground state has been detected in combustion environments ${ }^{53-55}$, the interstellar medium ${ }^{56,57}$ and under plasma conditions ${ }^{58}$. Reactions between $\mathrm{CH}$ and small unsaturated hydrocarbons and carbonyls can result in $\mathrm{CH}$ insertion to a $\pi$-bond through a cyclic intermediate (cycloaddition) followed by ring-opening ${ }^{51,59-62}$. This reaction has a negligible activation barrier, resulting in fast reaction rates. Isotopomer distribution experiments provide additional evidence for this mechanism through the reaction of deuterated methylidyne (CD) with ethylene and pyrrole ${ }^{50,59}$. $\mathrm{CH}$ is incorporated in the MACA mechanism, which has been demonstrated through the formation of indene from styrene ${ }^{37}$. Our group has also reported the formation of the methyltropyl radical and styrene from the discharge of toluene, an example of a six-membered ring converting to a seven-membered ring through $\mathrm{CH}$ insertion, with reformation of the aromatic ring upon decomposition ${ }^{63}$.

In an electrical discharge containing methane, both methyl radicals and $\mathrm{CH}$ will be present. Introducing $\mathrm{ACYN}$ thus allows the $\mathrm{CH}$ insertion reaction to compete with the methylation ring expansion reaction to generate phenalenyl radicals. But, these mechanisms will incorporate a different number of deuterium atoms if $\mathrm{CH}_{4}$ is substituted with $\mathrm{CD}_{4}$. In this work, we investigate the products of an electrical discharge containing methane and ACYN. We identify phenalenyl radical $\left(\mathrm{C}_{13} \mathrm{H}_{9}\right)$ as a product, and with perdeuterated methane detect a $\mathrm{C}_{13} \mathrm{H}_{8} \mathrm{D}$ radical identified to be phenalenyl-d1. No $\mathrm{C}_{13} \mathrm{H}_{7} \mathrm{D}_{2}$ radical is observed, which is strong evidence for $\mathrm{CH}$ cycloaddition 
as the dominant ring expansion mechanism. We propose that this reaction occurs in partnership with HACA to overcome the acenaphthylene bottleneck and lead to the generation of large PAHs.

\section{Results and Discussion}

The mass spectrum of an ACYN-containing argon beam (1\% $\mathrm{CH}_{4} / 99 \% \mathrm{Ar}$, Coregas) ionized by a $266 \mathrm{~nm}$ laser pulse is shown in Figure 2a. At this wavelength, species with an ionization energy $I E<9.32 \mathrm{eV}$ may be ionized through (resonant) 2-photon ionization (R2PI). The ACYN parent signal $\mathrm{C}_{12} \mathrm{H}_{8}(m / z 152)$ is minor compared to $m / z 154$. The $m / z 154$ signal corresponds to acenaphthene (ACN) $\mathrm{C}_{12} \mathrm{H}_{10}$, an impurity in the sample, that is resonance-enhanced at $266 \mathrm{~nm}$ $\left(\mathrm{S}_{2} \leftarrow \mathrm{S}_{0} \text { electronic transition }\right)^{64}$. Conversely, the parent $m / z 152$ signal is weaker than expected due to the short $(<16 \mathrm{ps})$ lifetime of the $\mathrm{ACYN}_{2}$ state $^{65}$. Peaks at $m / z 153$ and $\mathrm{m} / z 155$ are assigned to hydrogen atom loss and the ${ }^{13} \mathrm{C}$ isotopologue of $\mathrm{ACN}$, respectively. The species at $m / z 172$ is unidentified.

Figure $2 \mathrm{~b}$ shows the mass spectrum from ACYN, with a high voltage (HV) discharge of -1.05 $\mathrm{kV}$ striking the molecular beam expansion $2 \mathrm{~cm}$ after the pulsed nozzle. Two new groups of ions are generated by the HV discharge between $m / z 164-170$ and $m / z 178-184$, respectively. These two groups are subsequently spaced from the parent signal $(\mathrm{m} / z 152)$ by $12-18$ amu indicating the consecutive net addition of $\mathrm{CH}_{n}$, where $n=0-6$.

The species at $m / z 165$ is assigned to the phenalenyl radical, $\mathrm{C}_{13} \mathrm{H}_{9}$, resulting from net $\mathrm{CH}$ addition to ACYN. The peak at $m / z 166$ is assigned to $1 \mathrm{H}$-phenalene, $\mathrm{C}_{13} \mathrm{H}_{10}{ }^{48}$. The other significant ion signal in this cluster is at $m / z 168$, which corresponds to the addition of $\mathrm{CH}_{4}$ to $\mathrm{ACYN}$, resulting in a molecular formula of $\mathrm{C}_{13} \mathrm{H}_{12}$. A likely suspect is a species similar to $1 \mathrm{H}$-phenalene but with three consecutive, peripheral $\mathrm{sp}^{3}$ carbons (naphthalene chromophore). Additional minor peaks at $m / z 167$ and 169 are most likely a combination of ${ }^{13} \mathrm{C}$ isotopologues (of $\mathrm{m} / z 166$ and 168) and radical species.

To confirm the assignment of $m / z 165$, a tunable laser pulse was introduced to excite the 


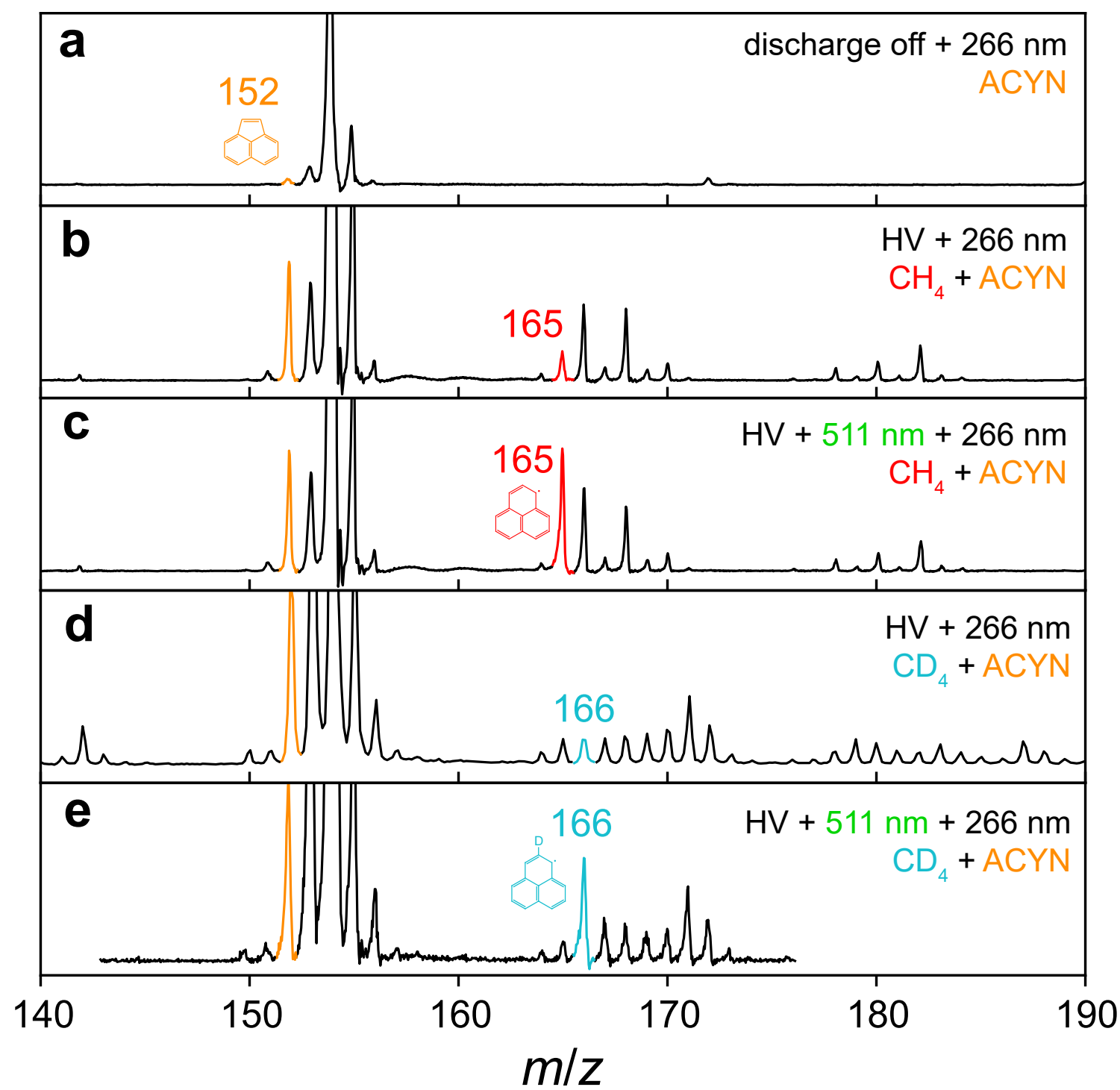

Figure 2: Mass spectra of products formed in high voltage (HV) electric discharge containing acenaphthylene and $\mathrm{CH}_{4} / \mathrm{CD}_{4}$. The parent ACYN $(m / z 152)$ is highlighted in yellow and the phenalenyl radical $(m / z 165)$ is highlighted in red. a) R2PI of ACYN at $266 \mathrm{~nm}$ without electric discharge. b) R2PI of products formed in electric discharge from ACYN $+\mathrm{CH}_{4}$ at $266 \mathrm{~nm}$. c) $\mathrm{R} 2 \mathrm{C} 2 \mathrm{PI}$ of products formed in electrical discharge from $\mathrm{ACYN}+\mathrm{CH}_{4}$. Excitation laser tuned to transition of phenalenyl at $19560 \mathrm{~cm}^{-1}(511 \mathrm{~nm})$ and preceding ionisation laser at $266 \mathrm{~nm}$ by $30 \mathrm{~ns}$. d) R2PI of products formed in electric discharge from ACYN $+\mathrm{CD}_{4}$ at $266 \mathrm{~nm}$. e) R2C2PI of products formed in electrical discharge from $\mathrm{ACYN}+\mathrm{CD}_{4}$. Excitation laser tuned to transition at $19565 \mathrm{~cm}^{-1}$ and preceding ionisation laser at $266 \mathrm{~nm}$ by $30 \mathrm{~ns}$. The 1d-phenalenyl radical $(\mathrm{m} / z$ 166) is highlighted in cyan.

radicals, preceding the ionizing laser pulse by $\sim 30 \mathrm{~ns}$. The wavelength of the excitation laser was scanned from $541-476 \mathrm{~nm}$ to measure the resonant 2-colour 2-photon ionisation (R2C2PI) 
spectrum for the $\mathrm{D}_{1} \leftarrow \mathrm{D}_{0}$ transition of the phenalenyl radical. Figure 3 shows a comparison to our previously reported R2C2PI spectrum ${ }^{49}$. The resonance-enhanced ion signal resulting from the excitation laser being tuned to the wavelength of the strongest vibronic transition is shown in Figure 2c.

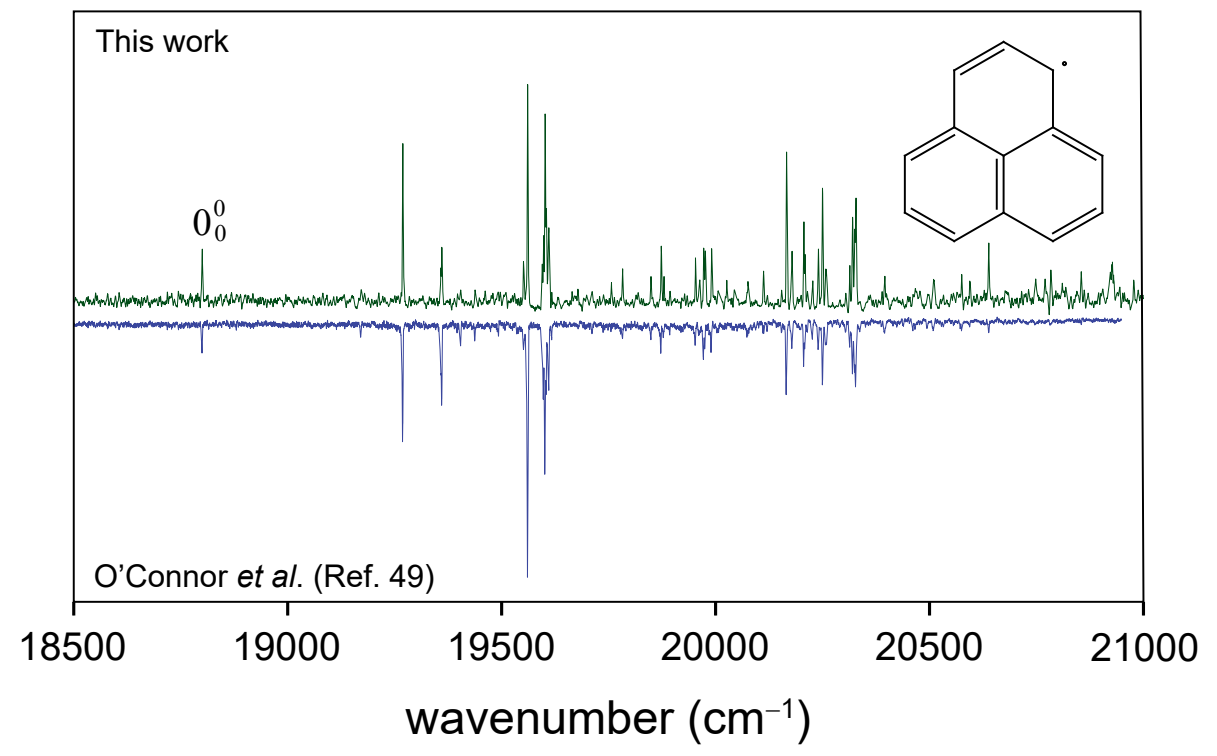

Figure 3: Resonant ionisation spectra of the phenalenyl radical $(m / z 165)$. Top: R2C2PI spectrum recorded in this work (green). Bottom: R2C2PI spectrum recorded by O'Connor et al. ${ }^{49}$ (blue).

The formation mechanism of the phenalenyl radical from ACYN was investigated by substituting perdeuterated methane, $\mathrm{CD}_{4}$, into the seeding gas mixture. The resultant mass spectrum from $266 \mathrm{~nm}$ ionization is shown in Figure $2 \mathrm{~d}$. As with the non-deuterated mass spectrum, there are two distinct mass peak clusters produced by $\mathrm{CD}_{n}$ and $\mathrm{C}_{2} \mathrm{D}_{n}$ addition to ACYN and ACN. Notably, the intensity of the deuterated mass peaks in these clusters is more evenly distributed across each mass and is no longer dominated by certain peaks, such was the case with $1 \mathrm{H}$-phenalene or $m / z 168$ in the non-deuterated mass spectrum. This suggests there are multiple contributing mechanisms involved in the production of these species, each incorporating a different number of hydrogens/deuteriums along the specific pathways.

The wavelength of the excitation laser was scanned around $511 \mathrm{~nm}$ to maximize the $\mathrm{m} / \mathrm{z}$ 166 signal. The resultant mass spectrum is displayed in Figure 2e. The resonance enhancement strongly suggests that the $m / z 166$ mass signal corresponds to the phenalenyl-d 1 radical, $\mathrm{C}_{13} \mathrm{H}_{8} \mathrm{D}$. 
To confirm this assignment, the excitation laser was scanned from $535 \mathrm{~nm}$ to $475 \mathrm{~nm}$. A comparison between the R2C2PI spectra of the phenalenyl radical and the species with $m / z 166$ is shown in Figure 4. Importantly, no other species produced a vibronic spectrum in this wavelength range. The strong resemblance to the spectrum of the phenalenyl radical confirms the assignment of $m / z 166$ to $\mathrm{C}_{13} \mathrm{H}_{8} \mathrm{D}$. Each peak (cluster) in the deuterated spectrum has been shifted to higher energy by $\sim 5 \mathrm{~cm}^{-1}$ compared to the original spectrum. Additional structure is also observed, with the deuterated spectrum dominated by a doublet-like splitting, as highlighted by the insets showing the regions around 18800 and $19560 \mathrm{~cm}^{-1}$.

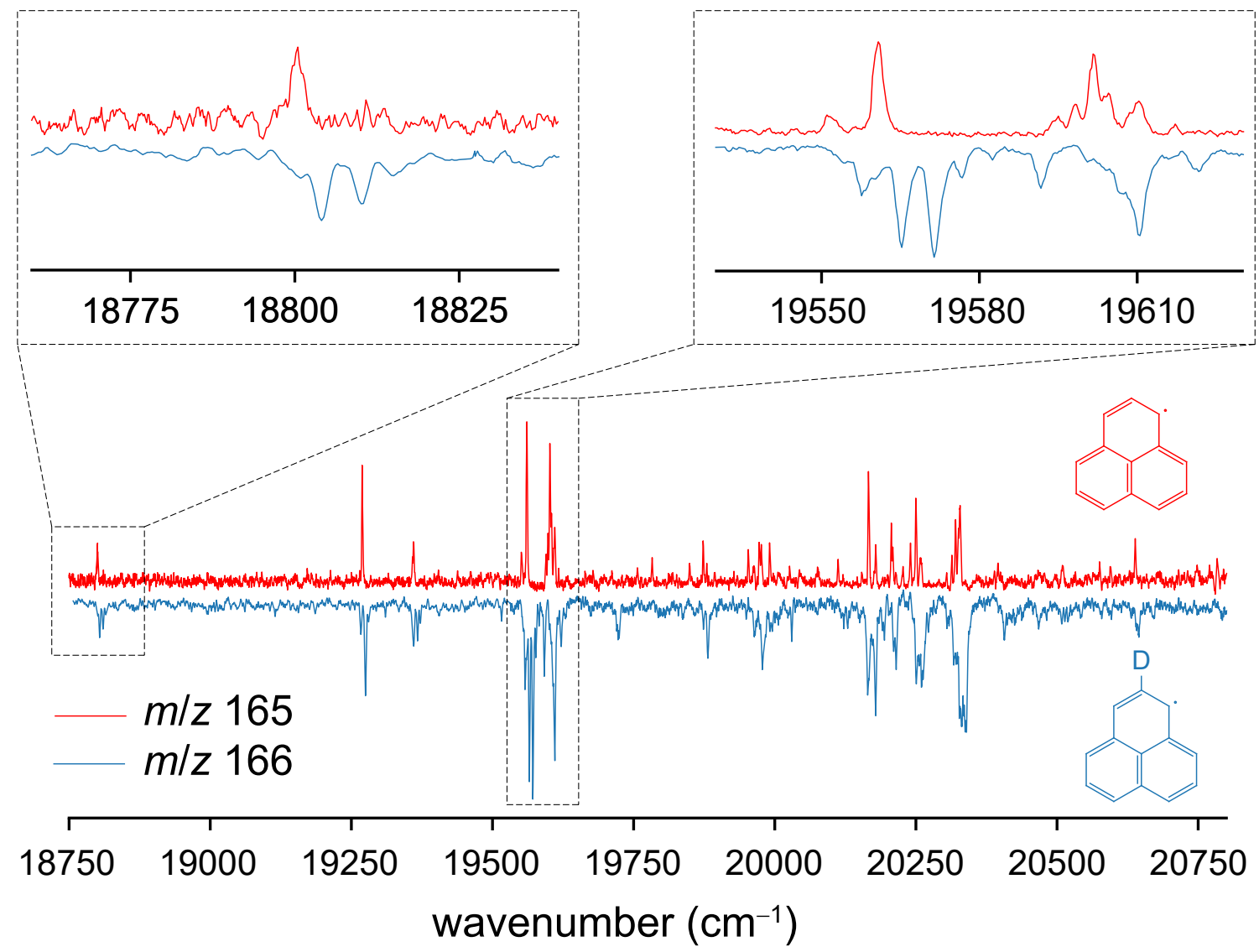

Figure 4: Top: A comparison of the resonant ionisation spectra of phenalenyl radical $\left(\mathrm{C}_{13} \mathrm{H}_{9}\right)$ (red) and deuterated phenalenyl radical $\left(\mathrm{C}_{13} \mathrm{H}_{8} \mathrm{D}\right)$ (blue). Top left: Origin band. Top right: Most intense bands in the spectrum.

It may be tempting to ascribe the splitting of the origin peak at $18800 \mathrm{~cm}^{-1}$ to two different deuteration sites: either on or off the $\mathrm{C}_{2}$ axes. However, the $\mathrm{D}_{1}$ state of phenalenyl is an $E^{\prime \prime} \otimes e^{\prime}$ Jahn-Teller problem. Briefly, the potential energy component of the Hamiltonian for the $1 E^{\prime \prime}$ state 
of phenalenyl can be written, in the diabatic basis,

$$
\mathbf{V}=\left[\begin{array}{cc}
\lambda \rho^{2}-k Q_{x}+\left(\delta Q_{x}\right) & +k Q_{y} \\
+k Q_{y} & \lambda \rho^{2}+k Q_{x}
\end{array}\right]
$$

where the rows and columns represent the two components of the degenerate electronic state, $\left|\mathcal{E}_{x}\right\rangle,\left|\mathcal{E}_{y}\right\rangle$. The symbols $Q_{x}$ and $Q_{y}$ are the components of a degenerate $e^{\prime}$ Jahn-Teller active mode, with $\rho^{2}=Q_{x}^{2}+Q_{y}^{2}$. The term in parentheses is discussed below. Diagonalization of this matrix $(\delta=0)$ generates the familiar Jahn-Teller conical intersection.

With a single deuterium atom there will be a zero-point energy difference between positive and negative distortions in, say, $Q_{x}$. This is quantum-induced symmetry-breaking, as observed in dihydroanthracenyl radicals. ${ }^{66}$ This effect is introduced by augmenting the linear term in at least one of the diagonal matrix elements. This tilts the potential to localize the minimum energy. ${ }^{67}$ A full account of this effect in asymmetrically deuterated cyclopentadienyl radicals is given in Reference 67.

In the basis of $\left|\mathcal{E}_{x, y}\right\rangle \otimes\left|v_{x}, v_{y}\right\rangle$ harmonic oscillator wavefunctions, $\left|\mathcal{E}_{x, y}\right\rangle \otimes\{|0,0\rangle,|1,0\rangle,|0,1\rangle\}$ which are eigenfunctions of the potential $V=\lambda \rho^{2}$, with eigenvalues $\left\{\epsilon_{0}, \epsilon_{1}, \epsilon_{1}\right\}$, the Hamiltonian may be written

$$
\mathbf{H}=\left[\begin{array}{cccccc}
\epsilon_{0} & -\beta^{\prime} & 0 & 0 & 0 & +\beta \\
-\beta^{\prime} & \epsilon_{1} & 0 & 0 & 0 & 0 \\
0 & 0 & \epsilon_{1} & +\beta & 0 & 0 \\
0 & 0 & +\beta & \epsilon_{0} & +\beta & 0 \\
0 & 0 & 0 & +\beta & \epsilon_{1} & 0 \\
+\beta & 0 & 0 & 0 & 0 & \epsilon_{1}
\end{array}\right]
$$

where $\left\langle 1\left|k Q_{x, y}\right| 0\right\rangle=\beta$ and $\beta^{\prime}=\beta(k-\delta) / k$. In the absence of zero-point energy effects, diagonalization of this matrix yields a degenerate vibrational ground state. However, the term $\delta$ lifts this degeneracy, resulting in a splitting, consistent with the experimental spectrum presented in Figure 4. An even more complicated pattern than is observed might be expected were there multi- 
ple isotopomers present in comparable quantities, in addition to the asymmetric Jahn-Teller effect. A complete assignment of the deuterated spectrum will be provided in a forthcoming publication.

This result provides insight into the formation mechanism of phenalenyl radical from ACYN. A mass shift from $m / z 165$ to 166 shows that only a single deuterium atom has been substituted into the phenalenyl radical. This is inconsistent with the methylation mechanism put forth by Porfiriev et. al. ${ }^{46}$, in which hydrogen abstraction from $\mathrm{ACYN}$ allows for $\mathrm{CH}_{3}\left(\mathrm{CD}_{3}\right)$ addition to the resultant radical site of the acenaphthyl radical. The methylated product may then follow several different pathways leading to the phenalenyl radical. However, each pathway in their proposed reaction scheme involves two hydrogen (deuterium) atoms from the $\mathrm{CH}_{3}\left(\mathrm{CD}_{3}\right)$ radical being present in the final product ${ }^{46}$. The deuterated reaction scheme for the methylation mechanism is shown in Figure 5a.

a

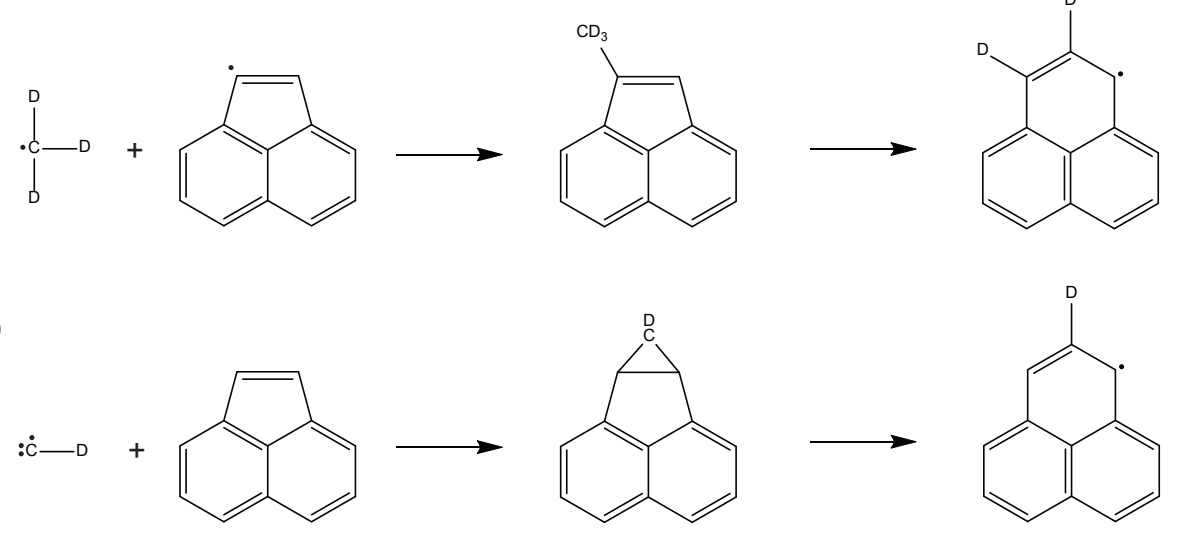

Figure 5: a) Methylation reaction scheme for formation of phenalenyl radical from 1-acenaphthyl radical $+\mathrm{CD}_{3}$, proposed by Porfiriev et. al. ${ }^{46}$ b) Cycloaddition reaction scheme for the formation of phenalenyl radical from acenaphthylene + CD .

Results from the present work are in agreement with the $\mathrm{CH}$ cycloaddition mechanism reported by Trevitt and Goulay ${ }^{62}$, where $\mathrm{CH}$ addition across a double bond allows for expansion of the fivemembered ring in ACYN to form one of the six-membered rings in phenalenyl. However, this process is also complicated by the potential for $\mathrm{CH}$ reaction at the six-membered rings in $\mathrm{ACYN}$, which one might expect to lead to tropyl- and benzyl-like structures. To investigate these aspects of the mechanism, we carried out a computational chemistry investigation.

Figure 6 shows the reaction scheme developed for phenalenyl formation from $\mathrm{CH}$ attack on the 
five-membered ACYN ring. The cycloadduct channel, depicted as the lower pathway, is formed via $\mathrm{CH}$ attack across the double bond of the five-membered ring and cuts through multiple pathways to directly form phenalenyl. By tracing the single added hydrogen/deuterium (shown in red) along this pathway we can deduce it will finish in the 2-position $\left(\mathrm{C}_{2 \mathrm{v}}\right)$. $\mathrm{CH}$ insertion into the $\mathrm{C}-\mathrm{H}$ bond of the five-membered ACYN ring may also occur, as depicted by the upper pathways. The resultant intermediate undergoes ring opening chemistry, generating phenalenyl either via a cycloadduct, or by direct insertion into the double bond. Tracing the hydrogen/deuterium along these pathways results in an ambiguous final position, either on or off the $\mathrm{C}_{2}$ axes. However, one isotopomer dominates the experimental spectra presented in Figure 4, so the reaction mechanism must have a propensity to favour one isotopomer over the other, consistent with the cycloaddition mechanism dominating over $\mathrm{CH}$ insertion.

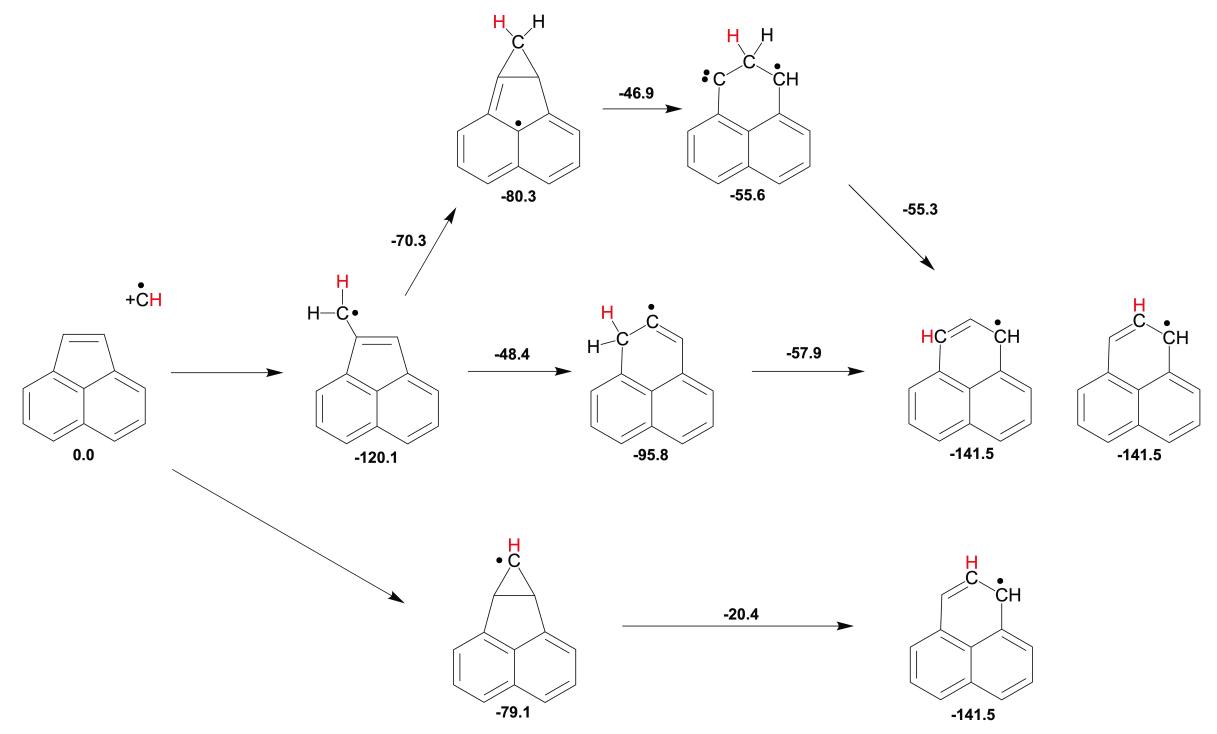

Figure 6: Reaction scheme for formation of phenalenyl from a $\mathrm{CH}$ attack on the five-membered ACYN ring. The single added hydrogen is shown in red. Calculations were performed at the G3X$\mathrm{K}$ level of theory. The energies are in $\mathrm{kcal} / \mathrm{mol}$.

As mentioned, $\mathrm{CH}$ reaction with ACYN may also proceed via attack at a six-membered ring, where cycloaddition and $\mathrm{C}-\mathrm{H}$ bond insertion are again both available. Figure 7 demonstrates that the cycloaddition process leads to a tropyl-like intermediate structure with no barrier. It may then rearrange to phenalenyl, with all barriers well below entrance. The formed tropyl-like intermediate 
undergoes an internal $\mathrm{H}$-atom transfer, a rate limiting step in the rearrangement to phenalenyl. Subsequently, a ring formation and cleavage mechanism occurs via a low energy barrier to finally produce phenalenyl. Following the added deuterium atom we see that both pathways depicted in Figure 7 result in a single isotopomer $\left(\mathrm{C}_{s}\right)$. To account for only one isotopomer being observed in the deuterated spectrum, therefore, cycloaddition must favour one of the cycloaddition mechanisms - attack at either the five or six-membered ring.

The $\mathrm{C}-\mathrm{H}$ bond insertion mechanism at the six membered ring is somewhat more complex, as there are three unique insertion sites, leading to three unique benzyl-like intermediates. One of these three processes is illustrated in Figure 8 with the others presented in the supplementary information $\dagger$. Again, formation of the initial resonantly stabilised radical (RSR) is barrierless and highly exothermic, providing it with significant excess vibrational energy to overcome subsequent barriers to rearrangement. Here this occurs via $\mathrm{CH}_{2}$ attack followed by insertion into the ring at either of the two adjacent carbons. A subsequent H-shift then returns the tropyl-like structure seen before, which will follow on to produce phenalenyl. As with insertion of a $\mathrm{C}-\mathrm{H}$ on the five membered ring, this process results in scrambling of $\mathrm{H}$ and $\mathrm{D}$ when $\mathrm{CD}$ initiates the reaction. The final isotopomer therefore can not be unique, again leading us to conclude that cycloaddition must be the dominant mechanism.

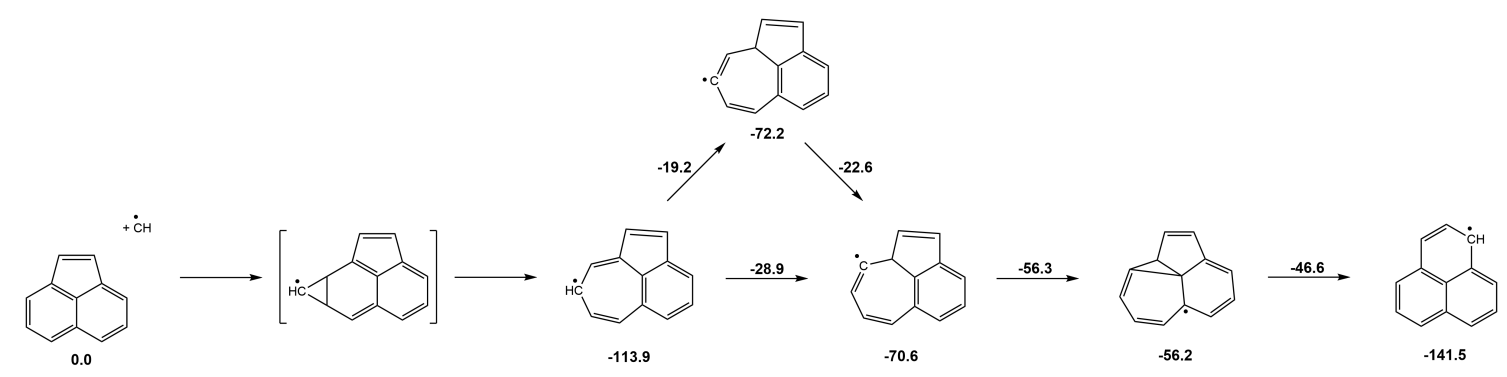

Figure 7: Formation of phenalenyl from a $\mathrm{CH}$ attack on the 6-membered ACYN ring, via a tropyllike intermediate RSR. Calculations were performed at the G3X-K level of theory. The energies are in $\mathrm{kcal} / \mathrm{mol}$.

In short, all roads lead to phenalenyl. The most straightforward route is via direct attack at the five-membered ring, and for the $C D$ reaction this produces a single $C_{2 v}$ isotopomer, which is consistent with our spectroscopic analysis. Cycloaddition and insertion at the six-membered rings 


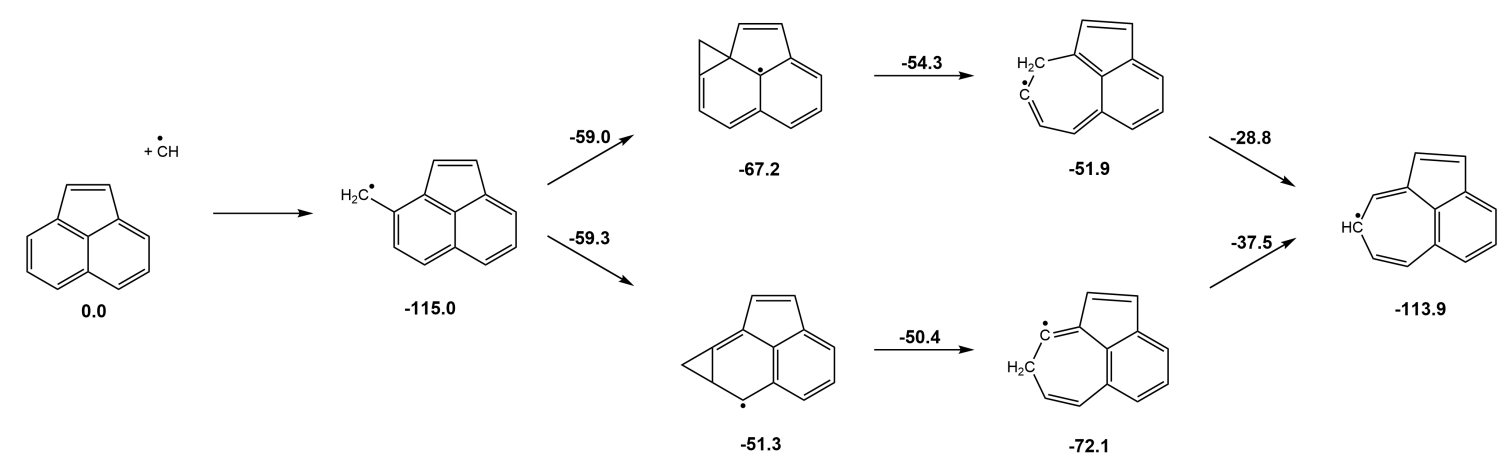

Figure 8: Reaction scheme for $\mathrm{CH}$ insertion, forming the tropyl-like RSR. Calculations were performed at the G3X-K level of theory. The energies are in $\mathrm{kcal} / \mathrm{mol}$.

makes tropyl- and benzyl-like RSRs, but in low pressure and energetic environments they will be able to rearrange to phenalenyl.

The $\mathrm{CH}$ cycloaddition mechanism working in conjunction with the HACA mechanism is a plausible explanation for the production of the multitude of large, planar PAHs formed in combustion processes. The HACA mechanism will predominantly generate a five-membered ring in the bay region of a $\mathrm{PAH}$. The $\mathrm{CH}$ cycloaddition mechanism will then cause ring expansion to form another six-membered ring, and the process can repeat itself. This subsequent combination of mechanisms can account for the formation of PAHs beyond the phenalenyl radical, such as pyrene, cyclopenta[cd]pyrene and the olympicenyl radical. The high concentrations of $\mathrm{CH}$ radicals in combustion environments and its detection in the interstellar medium further supports the plausibility of $\mathrm{CH}$ cycloaddition as a crucial component of the PAH formation mechanism ${ }^{53-57}$.

\section{Conclusions}

We have shown that the phenalenyl radical is produced as a discharge product of ACYN and methane. Substituting $\mathrm{CD}_{4}$ into the seeding gas mixture indicated that the dominant formation mechanism was $\mathrm{CH}$ cycloaddition. Calculations show that this reaction occurs through the insertion of $\mathrm{CH}$ across the $\mathrm{C}-\mathrm{C} \pi$-bond of the cyclopenta-fused ring of $\mathrm{ACYN}$, forming a threemembered ring, followed by ring-opening to generate the phenalenyl radical. This contradicts the suggestion that the reaction occurs by methylation ${ }^{46}$. The conversion of ACYN to the phenalenyl 
radical is an example of a five-membered ring expanding to a six-membered ring, which is of importance in combustion and interstellar chemistry, and the abundance of $\mathrm{CH}$ supports the proposed mechanism in these environments. The $\mathrm{CH}$ cycloaddition mechanism may work in tandem with the HACA mechanism to generate larger PAHs beyond the tricyclic phenalenyl radical.

\section{Experimental Details}

The apparatus used for R2C2PI and R2PI has been described previously in more detail ${ }^{68}$. A sample of $\mathrm{ACYN}$ is heated to $85^{\circ} \mathrm{C}$ and seeded in 5 bar of a methane/argon gas mixture(Coregas, $1 \%$ Methane/99\% Argon). At $85^{\circ} \mathrm{C}$ the concentration of ACYN in the beam is $\sim 0.018 \%$. The seeded gas mix is expanded into a differentially pumped vacuum chamber through a pulsed discharge nozzle (PDN). A voltage of $-1.05 \mathrm{kV}$ with a ballast resistance of $8 \mathrm{k} \Omega$ is applied to the outer electrode of the PDN for $100 \mu \mathrm{s}$, resulting in a strike which coincides with the expanding gas pulse.

Methane is incorporated into the backing gas mixture to provide an additional source of $\mathrm{CH}$. The discharge products are cooled through supersonic expansion and the central, coldest section of the molecular beam $(\sim 10 \mathrm{~K})$ is passed through a $2 \mathrm{~mm}$ diameter skimmer into a second differentially pumped chamber. The cold molecular beam is probed between two positively charged extraction plates of a Wiley-McLaren-type time-of-flight mass spectrometer (ToF-MS) using R2C2PI spectroscopy.

A Nd:YAG-pumped dye laser is used as an excitation laser to investigate the first electronic excited states of the phenalenyl radical. The excited radicals are subsequently ionized using the fourth harmonic $(266 \mathrm{~nm})$ of a second $\mathrm{Nd}$ :YAG laser, pulsing a few tens of nanoseconds after the excitation pulse. The resultant cations are orthogonally accelerated up the length of the ToF-MS and detected by a multichannel plate (MCP) which produces an electronic signal that is displayed on an oscilloscope. Custom-written LabView software is used to record the spectra and a wavemeter is used to calibrate the laser wavelengths. 


\section{Computational details}

Quantum chemical computations were performed using Gaussian-16 program ${ }^{69}$. M06-2X/6-31G(2df,p) level of theory and G3X-K were utilized to perform geometry optimizations and single point energy calculations, respectively. G3X-K predicts energies with mean errors of around $0.6 \mathrm{kcal} / \mathrm{mol}$, appropriate for thermochemical kinetics ${ }^{70}$. Frequency computations verified that all stationary points possess zero imaginary frequencies and one for all saddle points. Intrinsic reaction coordinate calculations confirm the connectivity of transition states.

\section{Acknowledgements}

This research was funded by the Australian Research Council (DP190103151). TWS is supported by the Australian Research Council Centre of Excellence in Exciton Science (CE170100026).

\section{References}

(1) Frenklach, M. Reaction mechanism of soot formation in flames. Phys. Chem. Chem. Phys. 2002, 4, 2028-2037.

(2) Wang, H. Formation of nascent soot and other condensed-phase materials in flames. P. Combust. Inst. 2011, 33, $41-67$.

(3) Faccinetto, A.; Irimiea, C.; Minutolo, P.; Commodo, M.; D’Anna, A.; Nuns, N.; Carpentier, Y.; Pirim, C.; Desgroux, P.; Focsa, C.; et al., Evidence on the formation of dimers of polycyclic aromatic hydrocarbons in a laminar diffusion flame. Commun. Chem. 2020, 3, 112.

(4) Henning, T.; Salama, F. Carbon in the Universe. Science 1998, 282, 2204-2210.

(5) Jäger, C.; Huisken, F.; Mutschke, H.; Jansa, I. L.; Henning, T. Formation of Polycyclic Aro- 
matic Hydrocarbons and Carbonaceous Solids in Gas-Phase Condensation Experiments. Astrophys. J. 2009, 696, 706-712.

(6) Draine, B. T.; Li, A. Infrared Emission from Interstellar Dust. I. Stochastic Heating of Small Grains. Astrophys. J. 2001, 551, 807-824.

(7) Draine, B. T.; Li, A. Infrared Emission from Interstellar Dust. IV. The Silicate-Graphite-PAH Model in the Post-Spitzer Era. Astrophys. J. 2007, 657, 810-837.

(8) Ramanathan, V.; Carmichael, G. Global and regional climate changes due to black carbon. Nat. Geosci. 2008, 1, 221-227.

(9) Finlayson-Pitts, B. J. Tropospheric Air Pollution: Ozone, Airborne Toxics, Polycyclic Aromatic Hydrocarbons, and Particles. Science 1997, 276, 1045-1051.

(10) Denissenko, M. F.; Pao, A.; Tang, M.-S.; Pfeifer, G. P. Preferential Formation of Benzo[a]pyrene Adducts at Lung Cancer Mutational Hotspots in P53. Science 1996, 274, 430-432.

(11) Tiwari, M.; Sahu, S.; Pandit, G. Distribution of PAHs in different compartment of creek ecosystem: Ecotoxicological concern and human health risk. Environ. Tox. Pharm. 2017, 50, $58-66$.

(12) Tielens, A. G. G. M. The molecular universe. Rev. Mod. Phys. 2013, 85, 1021-1081.

(13) Pech, C.; Joblin, C.; Boissel, P. The profiles of the aromatic infrared bands explained with molecular carriers. Astron. Astrophys. 2002, 388, 639-651.

(14) Tielens, A. Interstellar Polycyclic Aromatic Hydrocarbon Molecules. Annu. Rev. Astron. Astr. 2008, 46, 289-337.

(15) Dwek, E.; Arendt, R. G.; Fixsen, D. J.; Sodroski, T. J.; Odegard, N.; Weiland, J. L.; Reach, W. T.; Hauser, M. G.; Kelsall, T.; Moseley, S. H.; et al., Detection and Characteriza- 
tion of Cold Interstellar Dust and Polycyclic Aromatic Hydrocarbon Emission, from COBE Observations. Astrophys. J. 1997, 475, 565-579.

(16) D'Hendecourt, L.; Ehrenfreund, P. Spectroscopic properties of polycyclic aromatic hydrocarbons (PAHs) and astrophysical implications. Adv. Space Res. 1997, 19, 1023-1032.

(17) Cernicharo, J.; Heras, A. M.; Tielens, A. G. G. M.; Pardo, J. R.; Herpin, F.; Guélin, M.; Waters, L. B. F. M. Infrared Space Observatory's Discovery of $\mathrm{C}_{4} \mathrm{H}_{2}, \mathrm{C}_{6} \mathrm{H}_{2}$, and Benzene in CRL 618. Astrophys. J. 2001, 546, L123-L126.

(18) McGuire, B. A.; Burkhardt, A. M.; Kalenskii, S.; Shingledecker, C. N.; Remijan, A. J.; Herbst, E.; McCarthy, M. C. Detection of the aromatic molecule benzonitrile $\left(c-\mathrm{C}_{6} \mathrm{H}_{5} \mathrm{CN}\right)$ in the interstellar medium. Science 2018, 359, 202-205.

(19) Cami, J.; Bernard-Salas, J.; Peeters, E.; Malek, S. E. Detection of $\mathrm{C}_{60}$ and $\mathrm{C}_{70}$ in a Young Planetary Nebula. Science 2010, 329, 1180-1182.

(20) Campbell, E. K.; Holz, M.; Gerlich, D.; Maier, J. P. Laboratory confirmation of $\mathrm{C}_{60}^{+}$as the carrier of two diffuse interstellar bands. Nature 2015, 523, 322-323.

(21) Mcguire, B. A.; Loomis, R. A.; Burkhardt, A. M.; Lee, K. L. K.; Shingledecker, C. N.; Charnley, S. B.; Cooke, I. R.; Cordiner, M. A.; Herbst, E.; Kalenskii, S.; et al., Detection of two interstellar polycyclic aromatic hydrocarbons via spectral matched filtering. Science 2021, 371, 1265-1269.

(22) Frenklach, M.; Feigelson, E. D. Formation of Polycyclic Aromatic Hydrocarbons in Circumstellar Envelopes. Astrophys. J. 1989, 341, 372.

(23) Frenklach, M.; Clary, D. W.; Gardiner, W. C.; Stein, S. E. Detailed kinetic modeling of soot formation in shock-tube pyrolysis of acetylene. Symp. (Int.) Combust. 1985, 20, 887 - 901.

(24) Parker, D. S. N.; Kaiser, R. I.; Troy, T. P.; Ahmed, M. Hydrogen Abstraction/Acetylene Addition Revealed. Angew. Chem. Int. Ed. 2014, 53, 7740-7744. 
(25) Yang, T.; Troy, T. P.; Xu, B.; Kostko, O.; Ahmed, M.; Mebel, A. M.; Kaiser, R. I. HydrogenAbstraction/Acetylene-Addition Exposed. Angew. Chem. Int. Ed. 2016, 55, 14983-14987.

(26) Zhao, L.; Kaiser, R. I.; Xu, B.; Ablikim, U.; Ahmed, M.; Joshi, D.; Veber, G.; Fischer, F. R.; Mebel, A. M. Pyrene synthesis in circumstellar envelopes and its role in the formation of 2D nanostructures. Nat. Astron. 2018, 2, 413-419.

(27) Raj, A.; Prada, I. D. C.; Amer, A. A.; Chung, S. H. A reaction mechanism for gasoline surrogate fuels for large polycyclic aromatic hydrocarbons. Combust. Flame 2012, 159, 500 $-515$.

(28) Micelotta, E. R.,; Jones, A. P.,; Tielens, A. G. G. M., Polycyclic aromatic hydrocarbon processing in a hot gas. Astron. Astrophys. 2010, 510, A37.

(29) Micelotta, E. R.,; Jones, A. P.,; Tielens, A. G. G. M., Polycyclic aromatic hydrocarbon processing in interstellar shocks. Astron. Astrophys. 2010, 510, A36.

(30) Micelotta, E. R.,; Jones, A. P.,; Tielens, A. G. G. M., Polycyclic aromatic hydrocarbon processing by cosmic rays. Astron. Astrophys. 2011, 526, A52.

(31) Melius, C. F.; Colvin, M. E.; Marinov, N. M.; Pit, W. J.; Senkan, S. M. Reaction mechanisms in aromatic hydrocarbon formation involving the $\mathrm{C}_{5} \mathrm{H}_{5}$ cyclopentadienyl moiety. Symp. (Int.) Combust. 1996, 26, $685-692$.

(32) Miller, J. A.; Melius, C. F. Kinetic and thermodynamic issues in the formation of aromatic compounds in flames of aliphatic fuels. Combust. Flame 1992, 91, 21 - 39.

(33) Johansson, K. O.; Head-Gordon, M. P.; Schrader, P. E.; Wilson, K. R.; Michelsen, H. A. Resonance-stabilized hydrocarbon-radical chain reactions may explain soot inception and growth. Science 2018, 361, 997-1000.

(34) Siegmann, K.; Sattler, K. Formation mechanism for polycyclic aromatic hydrocarbons in methane flames. J. Chem. Phys. 2000, 112, 698-709. 
(35) Shukla, B.; Susa, A.; Miyoshi, A.; Koshi, M. Role of Phenyl Radicals in the Growth of Polycyclic Aromatic Hydrocarbons. J. Phys. Chem. A 2008, 112, 2362-2369.

(36) Shukla, B.; Miyoshi, A.; Koshi, M. Role of Methyl Radicals in the Growth of PAHs. J. Am. Soc. Mass Spectrom. 2010, 21, 534 - 544.

(37) Doddipatla, S.; Galimova, G. R.; Wei, H.; Thomas, A. M.; He, C.; Yang, Z.; Morozov, A. N.; Shingledecker, C. N.; Mebel, A. M.; Kaiser, R. I.; et al., Low-temperature gas-phase formation of indene in the interstellar medium. Sci. Adv. 2021, 7, eabd4044.

(38) Frenklach, M.; Mebel, A. M. On the mechanism of soot nucleation. Phys. Chem. Chem. Phys. 2020, 22, 5314-5331.

(39) Kislov, V. V.; Sadovnikov, A. I.; Mebel, A. M. Formation Mechanism of Polycyclic Aromatic Hydrocarbons beyond the Second Aromatic Ring. J. Phys. Chem. A 2013, 117, 4794-4816.

(40) Moskaleva, L.; Mebel, A.; Lin, M. The $\mathrm{CH}_{3}+\mathrm{C}_{5} \mathrm{H}_{5}$ reaction: A potential source of benzene at high temperatures. Symp. (Int.) Combust. 1996, 26, $521-526$.

(41) Sharma, S.; Green, W. H. Computed Rate Coefficients and Product Yields for $\mathrm{c}-\mathrm{C}_{5} \mathrm{H}_{5}+\mathrm{CH}_{3}$ $\rightarrow$ Products. J. Phys. Chem. A 2009, 113, 8871-8882.

(42) Mebel, A. M.; Georgievskii, Y.; Jasper, A. W.; Klippenstein, S. J. Pressure-dependent rate constants for PAH growth: formation of indene and its conversion to naphthalene. Faraday Disc. 2016, 195, 637-670.

(43) Jasper, A. W.; Hansen, N. Hydrogen-assisted isomerizations of fulvene to benzene and of larger cyclic aromatic hydrocarbons. Proc. Comb. Inst. 2013, 34, 279 - 287.

(44) Zhao, L.; Kaiser, R. I.; Lu, W.; Xu, B.; Ahmed, M.; Morozov, A. N.; Mebel, A. M.; Howlader, A. H.; Wnuk, S. F. Molecular mass growth through ring expansion in polycyclic aromatic hydrocarbons via radical-radical reactions. Nat. Commun. 2019, 10. 
(45) Mebel, A. M.; Landera, A.; Kaiser, R. I. Formation Mechanisms of Naphthalene and Indene: From the Interstellar Medium to Combustion Flames. J. Phys. Chem. A 2017, 121, 901-926.

(46) Porfiriev, D. P.; Azyazov, V. N.; Mebel, A. M. Conversion of acenaphthalene to phenalene via methylation: A theoretical study. Combust. Flame 2020, 213, $302-313$.

(47) Morita, Y.; Suzuki, S.; Sato, K.; Takui, T. Synthetic organic spin chemistry for structurally well-defined open-shell graphene fragments. Nat. Chem. 2011, 3, 197-204.

(48) O’Connor, G. D.; Chan, B.; Sanelli, J. A.; Cergol, K. M.; Dryza, V.; Payne, R. J.; Bieske, E. J.; Radom, L.; Schmidt, T. W. Hydrogen-adduction to open-shell graphene fragments: spectroscopy, thermochemistry and astrochemistry. Chem. Sci. 2017, 8, 1186-1194.

(49) O’Connor, G. D.; Troy, T. P.; Roberts, D. A.; Chalyavi, N.; Fückel, B.; Crossley, M. J.; Nauta, K.; Stanton, J. F.; Schmidt, T. W. Spectroscopy of the Free Phenalenyl Radical. J. Am. Chem. Soc. 2011, 133, 14554-14557.

(50) Soorkia, S.; Taatjes, C. A.; Osborn, D. L.; Selby, T. M.; Trevitt, A. J.; Wilson, K. R.; Leone, S. R. Direct detection of pyridine formation by the reaction of $\mathrm{CH}(\mathrm{CD})$ with pyrrole: a ring expansion reaction. Phys. Chem. Chem. Phys. 2010, 12, 8750.

(51) Caster, K. L.; Donnellan, Z. N.; Selby, T. M.; Goulay, F. Kinetic Investigations of the CH ( $\left.\mathrm{X}^{2} \Pi\right)$ Radical Reaction with Cyclopentadiene. J. Phys. Chem. A 2019, 123, 5692-5703.

(52) Caster, K. L.; Selby, T. M.; Osborn, D. L.; Le Picard, S. D.; Goulay, F. Product Detection of the $\mathrm{CH}\left(\mathrm{X}^{2} \Pi\right)$ Radical Reaction with Cyclopentadiene: A Novel Route to Benzene. J. Phys. Chem. A 2021, 125, 6927-6939.

(53) Love, N.; Parthasarathy, R. N.; Gollahalli, S. R. Concentration Measurements of CH and OH Radicals in Laminar Biofuel Flames. Int. J. Green Energy 2011, 8, 113-120. 
(54) Tinaut, F. V.; Reyes, M.; Giménez, B.; Pastor, J. V. Measurements of $\mathrm{OH}^{*}$ and $\mathrm{CH}^{*}$ Chemiluminescence in Premixed Flames in a Constant Volume Combustion Bomb under Autoignition Conditions. Energ. Fuel 2011, 25, 119-129.

(55) Zhang, T.; Guo, Q.; Liang, Q.; Dai, Z.; Yu, G. Distribution Characteristics of $\mathrm{OH}^{*}$, $\mathrm{CH}^{*}$, and $\mathrm{C}_{2} *$ Luminescence in $\mathrm{CH}_{4} / \mathrm{O}_{2}$ Co-flow Diffusion Flames. Energ. Fuel 2012, 26, 5503-5508.

(56) Gerin, M., et al. Interstellar $\mathrm{CH}$ absorption in the diffuse interstellar medium along the sightlines to G10.6-0.4 (W31C), W49N, and W51. Astron. Astrophys. 2010, 521, L16.

(57) Adams, W. S. Some Results with the COUDÉ Spectrograph of the Mount Wilson Observatory. Astrophys. J. 1941, 93, 11.

(58) Zhou, J.; Fisher, E. R. Surface Reactivity and Energetics of CH Radicals during Plasma Deposition of Hydrogenated Diamondlike Carbon Films. J. Phys. Chem. B 2006, 110, 2191121919.

(59) Goulay, F.; Trevitt, A. J.; Meloni, G.; Selby, T. M.; Osborn, D. L.; Taatjes, C. A.; Vereecken, L.; Leone, S. R. Cyclic Versus Linear Isomers Produced by Reaction of the Methylidyne Radical (CH) with Small Unsaturated Hydrocarbons. J. Am. Chem. Soc. 2009, $131,993-1005$.

(60) Trevitt, A. J.; Prendergast, M. B.; Goulay, F.; Savee, J. D.; Osborn, D. L.; Taatjes, C. A.; Leone, S. R. Product Branching Fractions of the $\mathrm{CH}+$ Propene Reaction from Synchrotron Photoionization Mass Spectrometry. J. Phys. Chem. A 2013, 117, 6450-6457.

(61) Goulay, F.; Trevitt, A. J.; Savee, J. D.; Bouwman, J.; Osborn, D. L.; Taatjes, C. A.; Wilson, K. R.; Leone, S. R. Product Detection of the CH Radical Reaction with Acetaldehyde. J. Phys. Chem. A 2012, 116, 6091-6106.

(62) Trevitt, A. J.; Goulay, F. Insights into gas-phase reaction mechanisms of small carbon radicals using isomer-resolved product detection. Phys. Chem. Chem. Phys. 2016, 18, 5867-5882. 
(63) Reilly, N. J.; Da Silva, G.; Wilcox, C. M.; Ge, Z.; Kokkin, D. L.; Troy, T. P.; Nauta, K.; Kable, S. H.; Mccarthy, M. C.; Schmidt, T. W.; et al., Interconversion of Methyltropyl and Xylyl Radicals: A Pathway Unavailable to the Benzyl-Tropyl Rearrangement. J. Phys. Chem. A 2018, 122, 1261-1269.

(64) Swiderek, P.; Hohlneicher, G. Comment on the Low Lying Singlet States of Acenaphthene. Ber. Bunsenges. Phys. Chem. 1991, 95, 1683-1684.

(65) Flock, M.; Herbert, M.-P.; Fischer, I. The excited-state structure and photophysics of isolated acenaphthylene. Chem. Phys. 2018, 515, 744-749, Ultrafast Photoinduced Processes in Polyatomic Molecules:Electronic Structure, Dynamics and Spectroscopy (Dedicated to Wolfgang Domcke on the occasion of his 70th birthday).

(66) Krechkivska, O.; Wilcox, C. M.; Nauta, K.; Kable, S. H.; Schmidt, T. W. Quantum-Induced Symmetry Breaking in the Deuterated Dihydroanthracenyl Radical. J. Phys. Chem. A 2019, $123,6711-6719$.

(67) Yu, L.; Cullin, D. W.; Williamson, J. M.; Miller, T. A. High resolution laser spectroscopy of asymmetrically deuterated cyclopentadienyl radicals: A study of vibronic degeneracy resolution and Jahn-Teller distortion. J. Chem. Phys. 1993, 98, 2682-2698.

(68) Reilly, N. J.; Nakajima, M.; Troy, T. P.; Chalyavi, N.; Duncan, K. A.; Nauta, K.; Kable, S. H.; Schmidt, T. W. Spectroscopic Identification of the Resonance-Stabilized cis- and trans-1Vinylpropargyl Radicals. J. Am. Chem. Soc. 2009, 131, 13423-13429.

(69) Frisch, M. J. et al. Gaussian16 Revision C.01. 2016; Gaussian Inc. Wallingford CT.

(70) da Silva, G. G3X-K theory: A composite theoretical method for thermochemical kinetics. Chem. Phys. Lett. 2013, 558, 109-113. 